\title{
Monte Carlo simulation of MPPC photosensors for the T2K experiment
}

\author{
Martin Haigh* \\ University of Warwick, CV4 7AL Coventry, UK \\ E-mail: m.d.haigh@warwick.ac.uk
}

\author{
Antonin Vacheret \\ Imperial College London, SW7 2AZ London, UK \\ E-mail: a.vachereteimperial.ac.uk
}

\section{Fabrice Retière}

TRIUMF, 4004 Wesbrook Mall, Vancouver, BC, V6T 2A3, Canada

E-mail: fretiere@triumf.ca

\section{Thomas Lindner and Scott Oser}

University of British Columbia, 6224 Agricultural Road, Vancouver, BC, V6T 1Z1, Canada

E-mail: lindner@phas.ubc.ca, oser@phas.ubc.ca

\begin{abstract}
A Monte Carlo model of the MPPC photosensor has been developed by the T2K-ND280 collaboration, forming part of the detector readout chain simulation. This model incorporates all device features, including dark noise, crosstalk, afterpulsing and saturation, and also includes recovery effects specific to the ND280 front-end readout circuit. Simulation parameters have been tuned using the results of characterization measurements made by the collaboration. This talk outlines the framework and physics models used in the simulation. Results from the Monte Carlo are compared to data.
\end{abstract}

International Workshop on New Photon Detectors

June 24-26, 2009

Shinshu University Matsumoto Japan

\footnotetext{
* Speaker.
} 


\section{Introduction}

$\mathrm{T} 2 \mathrm{~K}[1]$ is a long-baseline accelerator neutrino experiment due to turn on at the end of 2009. A beam of neutrinos will be produced by the J-PARC proton synchrotron at Tokai, and the Super-K water Cerenkov detector and a suite of near detectors will be used to make a precision measurement of the mixing angle $\theta_{13}$. The main near detectors, INGRID and ND280[2], consist mostly of scintillator bars, with light channelled out along wavelength-shifting fibres and read out using MPPC photosensors. The MPPC[3][4] is a multi-pixel Geiger-mode APD (GM-APD) manufactured by Hamamatsu; these devices consist of an electrically parallel array of diode junctions, operated above their reverse breakdown voltage $V_{\mathrm{bd}}$, in series with quenching resistors, on a single semiconductor wafer. Because a single freed carrier in a pixel produces a Geiger-mode avalanche (arrested by the quenching resistor, which limits the flow of charge off the pixel), these devices have comparable gain to PMTs, while satisfying our requirements for compactness and insensitivity to magnetic fields. The active area of the devices used by T2K is $1.3 \times 1.3 \mathrm{~mm}^{2}$ with a pixel pitch of $0.05 \mathrm{~mm}$. The ND280 detector will use just over 40,000 MPPCs.

Considerable work has been done by $\mathrm{T} 2 \mathrm{~K}$ collaborators to characterize the MPPCs used in the experiment. The data collected has enabled the development of empirical models which quantitatively describe device behaviour. A Monte Carlo simulation of the MPPC, based on these models, has been developed; this forms part of the full ND280 MC, and can also be used to test the models used to describe individual device features.

\section{Description of the simulation}

The simulation consists of a set of models describing device features, and a procedural framework to initialize the models, process incoming photon data, control the simulation and output the result. All empirically important device features are modelled, including dark noise (DN), crosstalk (CT), afterpulsing (AP) and recovery effects. The device is modelled as a finite 2-dimensional array of pixels, so saturation is also included. Parameters which vary with the voltage above breakdown (overvoltage) are modelled as linear or quadratic functions.

The simulation is written in C++ and is integrated into the ND280 offline software framework; however a standalone version has also been developed which requires only the GSL libraries[5]. It is intended that this version be released for wider use in the near future.

\subsection{Procedural framework}

The framework of the simulation is based around a list of "free carriers", or potential pixel triggers. These are not always directly equivalent to free charges in the semiconductor, but can be anything which can cause an avalanche, i.e. an incident photon, thermal electron/hole, crosstalk photon or trapped charge (for afterpulsing). This list is initially populated by a set of incoming photons which must be passed to the simulation as input; the simulation itself adds thermal noise triggers to this list. The simulation then steps through the list of carriers in time order, using the physics models to decide whether the carrier triggers an avalanche; if so, the overvoltage of the fired pixel is set to zero and an avalanche is added to an output list, and a check is done to see 
whether CT/AP should be generated. Any CT/AP triggers are added to the list of carriers, to be processed in correct time order.

This time-ordered framework allows cascades of CT/AP to be dealt with simply and naturally, since any secondary noise effects will be calculated when the primary CT/AP trigger is processed. It also makes it easy to integrate voltage recovery of pixels into the simulation - the recovery model recalculates all pixel voltages before dealing with each carrier, and the only required variables to do the recovery calculation are the previous pixel voltages and the time elapsed since the last trigger. The only MPPC state variables used by the simulation are the carrier list and pixel voltages, though the ND280-specific pixel recovery model also stores data about the state of the external electronics.

\subsection{Physics models}

\subsubsection{Dark noise}

The rate of dark noise avalanches, due to thermally generated free carriers in the semiconductor, has been measured[6] to be linear in device overvoltage; a linear parametrization is therefore used in the simulation. It should be noted that noise rates at a given overvoltage vary by a factor $\sim 2$ between nominally identical MPPCs, so must be measured for every device if dark noise is expected to be an issue.

Dark pulses are generated before starting each simulation event, using a Poisson distribution with mean equal to the expected number of pulses in the period simulated. The pulses are distributed uniformly over the device pixels and over the simulation period. The dark rate used to generate the pulses is that calculated for the nominal applied bias voltage $\left(D N R\left(V_{\text {nom }}\right)\right)$; recovery effects are accounted for when the thermal carrier is processed, by only generating an avalanche with probability $D N R\left(V_{\text {pix }}(t)\right) / D N R\left(V_{\text {nom }}\right)$, i.e. the ratio of the dark rate at the pixel voltage to that at the nominal voltage. This prescription mathematically holds as long as the used recovery model does not allow a pixel voltage to rise above the nominal value.

\subsubsection{PDE}

The photon detection efficiency (PDE) of a photosensor is the probability that an incoming photon will be collected and converted to an output signal. For a GM-APD, this is the product of the geometrical efficiency (proportion of area which is active), quantum efficiency (probability to collect the photon), and Geiger avalanche probability. There is a strong voltage dependence mainly via the Geiger probability, and also a dependence on the wavelength of the incoming light. Wavelength dependence of the PDE is not currently modelled in the simulation, since the light important for the ND280 application is received in a single well-defined wavelength range from wavelength-shifting fibres. Overvoltage dependence is linear in the simulation, though detailed results, from the Institute for Nuclear Research in Moscow and other institutions[6][7], suggest that the parametrization needs to be made more sophisticated.

Procedurally, the application of the PDE model is very simple. All incident photons are stored in the carrier list, but only trigger an avalanche with probability $\operatorname{PDE}\left(V_{\text {pix }}(t)\right)$.

\subsubsection{Afterpulsing}

Afterpulsing is the name applied to delayed secondary pulses in the same pixel as an initial trigger, due to the trapping of charge carriers on impurities during an avalanche. This process can 


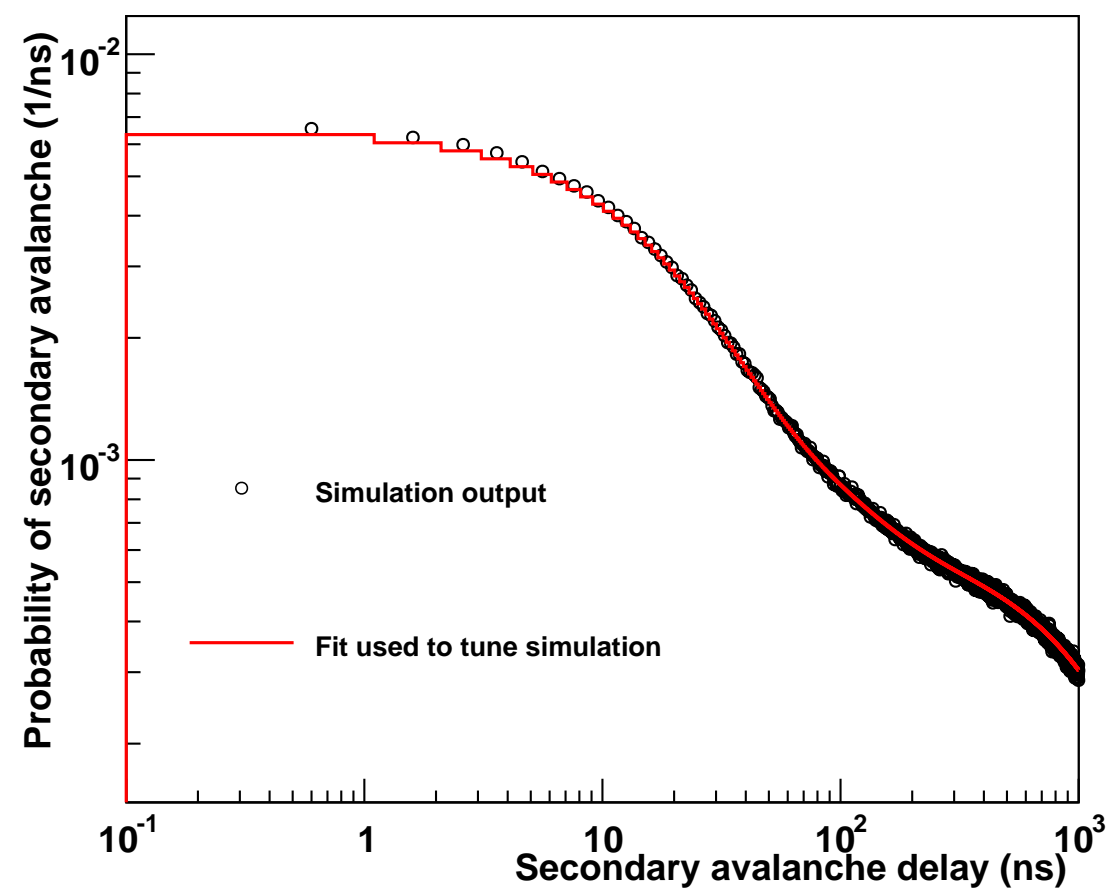

Figure 1: Time distribution of secondary pulses after a trigger pulse, for data and simulation.

be studied by measuring the distribution of time delays between an initial trigger pulse and the next observed pulse. The latter will be due to either uncorrelated thermal noise or afterpulsing, and the distribution can be fitted to a hypothesis for the afterpulse probability and time distribution.

Data of this kind taken at TRIUMF[6] supports a model where there are two charge trapping modes, the number of trapped charges of each type follows a Poisson distribution, and the time distribution for each type of afterpulse is an exponential $e^{-t / \tau_{i}}$, with a different time constant $\tau_{i}$ for each mode. The time constants are more-or-less independent of overvoltage, and are $\tau_{\mathrm{S}}=17.7 \mathrm{~ns}$, $\tau_{\mathrm{L}}=70.9 \mathrm{~ns}$. The mean number of trapped charges is measured to be $0.043 \times\left(V_{\text {pix }}-V_{\mathrm{bd}}\right)^{2}$ for each type.

The simulation uses the parameters measured at TRIUMF to determine how many short and long afterpulses are to be inserted into the carrier list from an initial pulse. The pulses are inserted at a time determined by the exponential distributions. The afterpulse probability is thus accounted for at the time the carrier is created, and so the carrier triggers an avalanche with a probability of unity. The distribution of secondary pulse delays is shown for data and simulation in figure 1 .

\subsubsection{Crosstalk}

Crosstalk is the process whereby a photon emitted during avalanche multiplication causes an almost simultaneous secondary avalanche in a neighbouring pixel. The probability of crosstalk is expected to vary with the position of the primary pixel, since pixels near the edge of the array have fewer neighbours. Data has been taken at Imperial College London using a laser focused on 


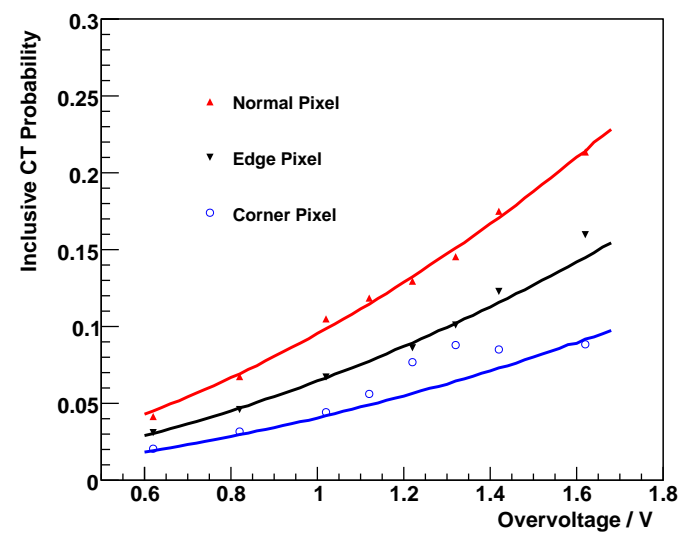

(a) Inclusive probability

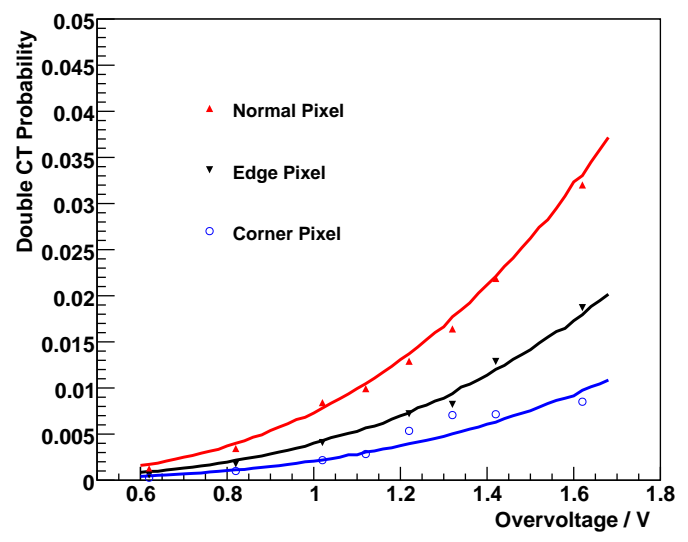

(b) Double CT probability

Figure 2: Data/MC comparison for inclusive and double crosstalk probabilities, for normal, edge and corner pixels. Data are shown as points; the model predictions are shown as corresponding-coloured lines.

a single pixel, yielding the probability of getting 1,2 and 3 crosstalks from a primary pulse, for "normal", edge and corner pixels, as a function of overvoltage. Different probabilities are observed for the three pixel positions as expected.

The simulation uses a simple crosstalk model with three tunable parameters. In the model, each avalanche can only produce a single crosstalk, but further crosstalks can be caused by cascade. The probability of crosstalk varies quadratically with the overvoltage of the primary pixel, $P_{\mathrm{CT}}=a\left(V-V_{\mathrm{bd}}\right)+b\left(V-V_{\mathrm{bd}}\right)^{2}$, and is tuned to match the inclusive CT probability for a "normal" pixel; the fitted values for the quadratic coefficients are $a=0.0356 \mathrm{~V}^{-1}, b=0.0598 \mathrm{~V}^{-2}$. The secondary pixel is chosen randomly in a $5 \times 5$ grid around the primary, with each pixel weighted by a factor $e^{-r / c}$, where $r$ is the distance from primary to secondary pixel. Lower CT for edge pixels is modelled since some of the neighbours may be off the edge of the device. With the range, $c$, set to $0.4 \times$ pixel pitch, the data is matched quite well for all pixel positions. The data and model results are compared in figure 2 .

\subsubsection{Gain}

The output of the simulation is a list of avalanches with start times and charge values (normalised to the gain at the operating voltage). The charge for each pulse is smeared using a Gaussian; the smearing width is obtained from histogrammed charge data (see figure 5 below) by comparing the widths of the zeroth (no pixels fired) and first peaks. If the voltage on the pixel is lower than the applied bias voltage, then the output charge is proportionately lower. The simulation outputs only a list of pulses, not complete waveforms.

\subsubsection{Recovery}

Because each MPPC pixel contains a quenching resistor in series with the junction, the voltage across the pixel takes a finite time to recover once it has fired. The intrinsic pixel time constant $\tau_{\mathrm{I}}=C_{\text {pix }} R_{\text {pix }}$ is $13.5 \mathrm{~ns}$ for a pixel capacitance $C_{\text {pix }}=90 \mathrm{fF}$, and resistance $R_{\text {pix }}=150 \mathrm{k} \Omega$. This is 


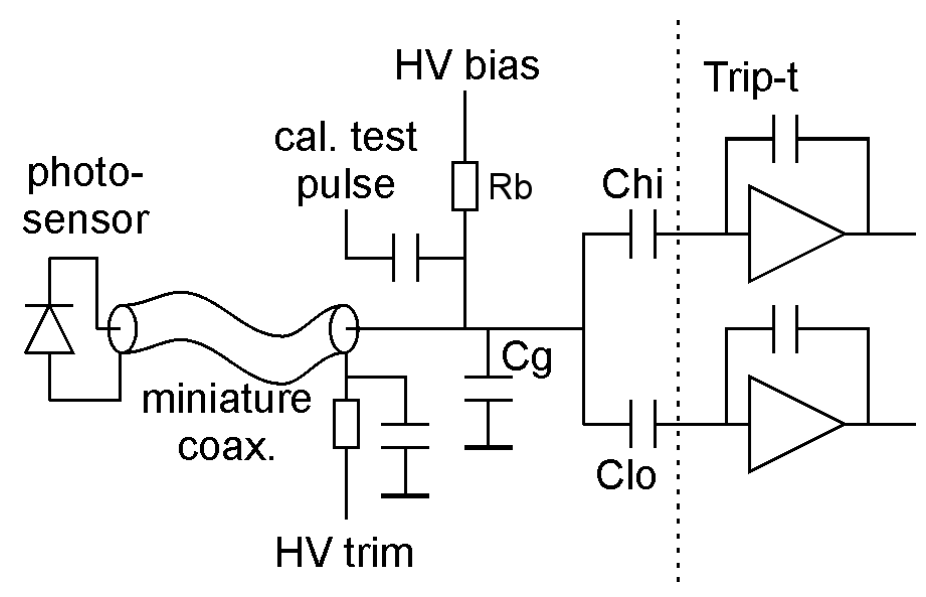

Figure 3: Schematic of the interface between the ND280 front-end board and the MPPC.

the pixel recovery time for an MPPC connected to a voltage source. The actual recovery behaviour will depend on the details of the readout circuit; in particular, if the current onto the device is limited, then some internal sharing of charge between the pixels is expected to occur. The recovery element of the simulation is separated into its own class, with an abstract base, so that it is relatively simple to implement a new recovery model for a specific readout circuit. At low light levels, recovery only has a significant effect on afterpulsing, but it is very important for modelling of saturation behaviour.

The front-end board (TFB) used in ND280[8] is based on the Trip-t ASIC chip developed for D0[9]. Two amplifier channels, with different gains, are used for each MPPC, in order to obtain the required dynamic range; charge is divided between the amplifiers according to their coupling capacitors $C_{\mathrm{lo}}$ and $C_{\mathrm{hi}}$. A single $\mathrm{HV}$ rail is used to bias all devices, but a variable trim voltage can be applied to the ground pin of each device in order to compensate for differences in breakdown voltage. A schematic of the interface between MPPC and amplifier is shown in figure 3.

A SPICE model has been built to simulate the electronics interface. The MPPC is modelled as $N_{\text {pix }} \mathrm{RC}$ series circuits in parallel; a current source pulses some of the pixels at $t=0$, draining them to zero overvoltage, to simulate some of the pixels firing. The main circuit features which affect recovery are the resistor $R_{\mathrm{b}}=100 \mathrm{k} \Omega$ between the bias source and device, and the capacitor $C_{\mathrm{g}}=330 \mathrm{pF}$. A small resistor $R_{\mathrm{g}}=50 \Omega$ (not shown on the schematic) separates the MPPC from $C_{\mathrm{g}}$. Transient effects occur when the amplifiers are reset.

Since the bias voltage source is behind a large resistance, the short-time recovery behaviour of the device can be modelled by ignoring it and considering only the MPPC and local capacitor $C_{\mathrm{g}}$. This circuit can be solved analytically and gives, for the pixel voltages $V_{i}(t)$

$$
\begin{array}{r}
V_{i}(t)=V_{i}(0) e^{-t / \tau_{\mathrm{I}}}+\left(V_{\mathrm{g}}(0)-\frac{A \tau^{\prime}}{C_{\mathrm{g}}}\right)\left(1-e^{-t / \tau_{\mathrm{I}}}\right)+\frac{A\left(\tau^{\prime} / C_{\mathrm{g}}-R_{\mathrm{g}}\right)}{1-\tau_{\mathrm{I}} / \tau^{\prime}}\left(e^{-t / \tau^{\prime}}-e^{-t / \tau_{\mathrm{I}}}\right) \\
\text { where } \tau_{\mathrm{I}}=R_{\text {pix }} C_{\text {pix }}, \tau^{\prime}=\frac{R_{\text {pix }} / N_{\text {pix }}+R_{\mathrm{g}}}{1 / C_{\mathrm{g}}+1 / C_{\text {pix }} N_{\text {pix }}}, A=\frac{V_{g}(0)-\sum_{i} V_{i}(0) / N_{\text {pix }}}{R_{\text {pix }} / N_{\text {pix }}+R_{\mathrm{g}}}
\end{array}
$$

and $V_{\mathrm{g}}$ is the voltage across the capacitor $C_{\mathrm{g}}$. For our component values, the last term is small, so the pixels still recover almost exponentially with a time constant $\sim \tau_{\mathrm{I}}$; the voltage converged upon 


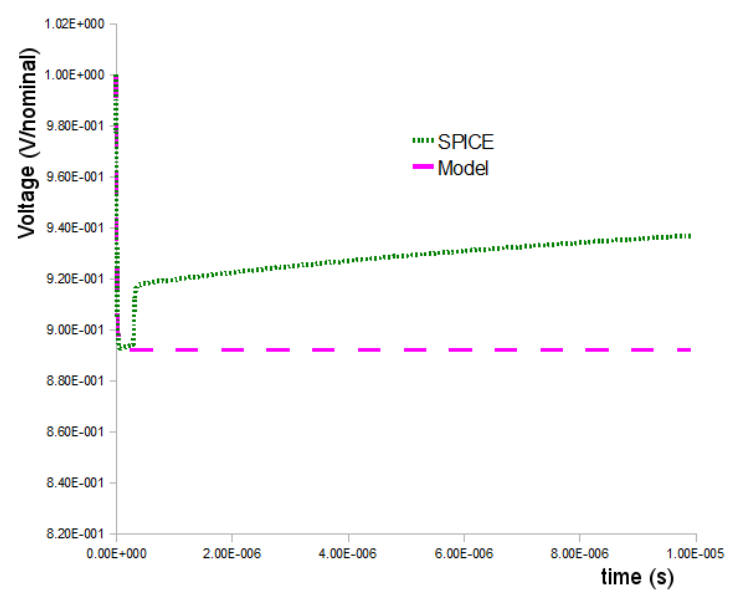

(a) Full scale

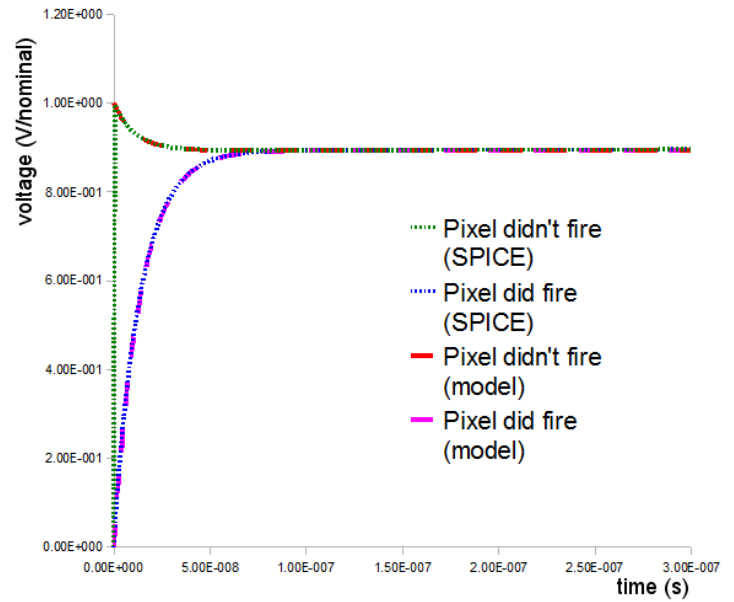

(b) Zoomed into short-time region

Figure 4: Comparison of the SPICE front-end board model with an analytic "local capacitor" model.

is a capacitance-weighted average of the initial voltages across the pixels and across $C_{\mathrm{g}}$. For large signals, this charge sharing can deplete the unfired pixels significantly.

It can be seen from figure 4 that this model agrees well with the full SPICE simulation for short times. For longer times, the two models disagree, since transient effects, and recharging from the voltage source, are not included in the analytic model. Modelling sensor response within a single bunch period (540 ns) is the main ND280 application for the Monte-Carlo, so the model meets our requirements.

\section{Results}

\subsection{Low light data comparison}

The ND280 electronics outputs an integrated charge measurement for each amplification cycle. Histogramming these measurements at low light level gives a series of peaks corresponding to different numbers of pixels firing in the MPPC. Data taken using this readout system is compared with the corresponding simulation output in figure 5, for low light conditions. Excellent agreement is seen between simulation and data over a range of bias voltages. It is likely that the agreement can be improved further with the implementation of a more sophisticated PDE model.

\subsection{Saturation behaviour}

The simulation has also been used to predict the response of the device to large signals. Saturation is expected since the device has a finite number of pixels and a non-zero recovery time. The response curve should depend on the time distribution of the incoming light signal, since for longer pulses then the pixels are able to recover significant voltage during the pulse, and may therefore be triggered more than once. Even for very fast pulses, pixels can be fired later by afterpulsing. Because not all triggers are simultaneous, it is possible for the total response (in pixel charges) to be higher than the number of pixels on the device. Because pixel recovery effects are important, saturation behaviour is expected to depend on the readout circuit used. 

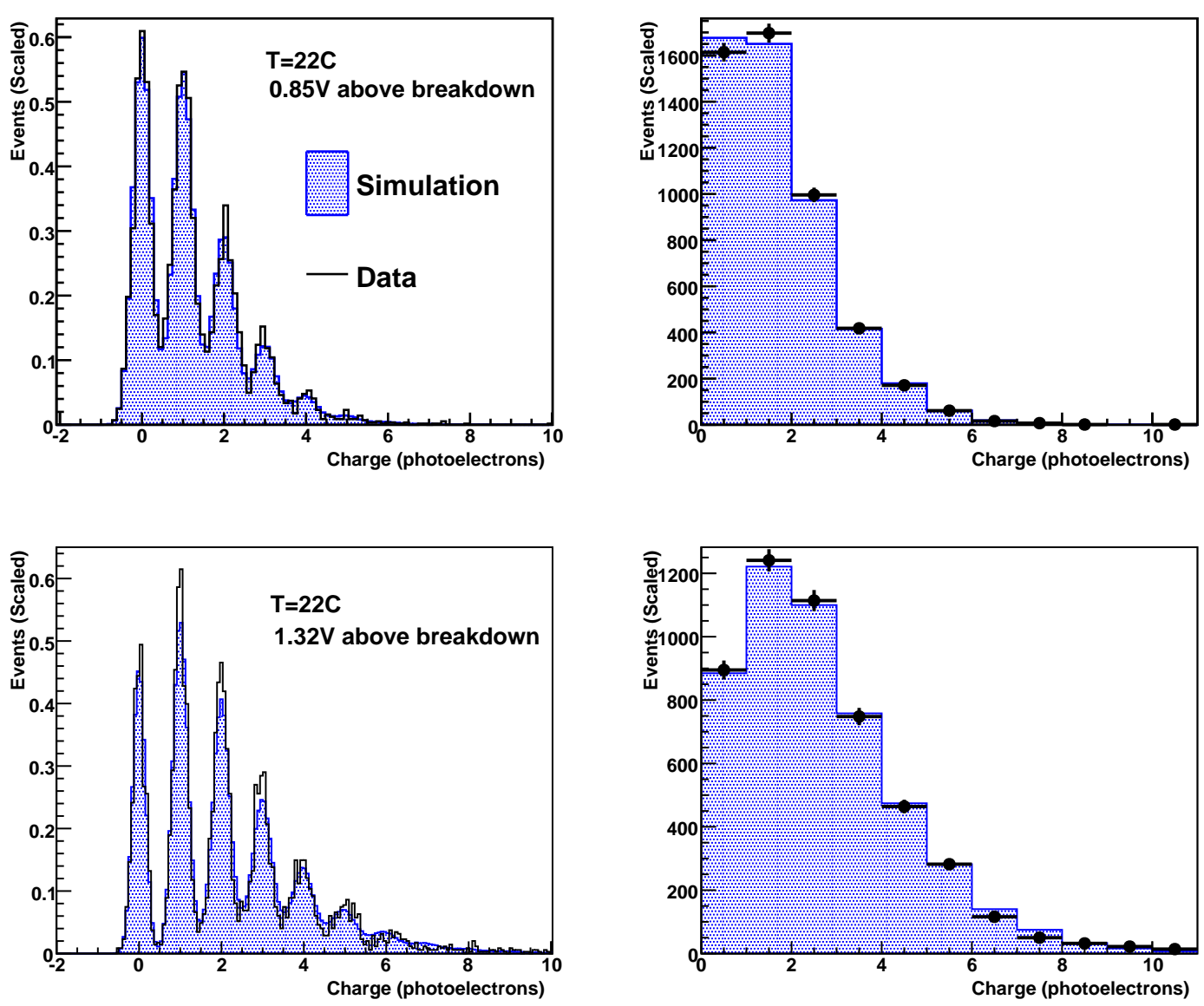

Figure 5: Comparison of simulation and data charge spectra. The device breakdown voltage was $69.77 \mathrm{~V}$. The right-hand plots are rebinned versions of the left-hand ones, showing the total number of events for each peak.

Simulated response curves, for an overvoltage of $1.32 \mathrm{~V}$, are shown in figure 6. The predicted saturation behaviour varies with the recovery model used and the incoming light distribution as expected; in particular, the response of the TFB electronics is lower than for a voltage source, since the total charge available from the capacitor $C_{\mathrm{g}}$ is limited. The collection of real data to test the $\mathrm{MC}$ prediction is now underway.

\section{Conclusion}

An empirically-driven Monte Carlo model for MPPC simulation has been developed by ND280 collaborators. It includes all relevant noise effects, and a recovery model based on a detailed study of the ND280 readout electronics. The simulation agrees well with data for a range of operating voltages, and has also been used to predict the response of the MPPC to large light signals. 


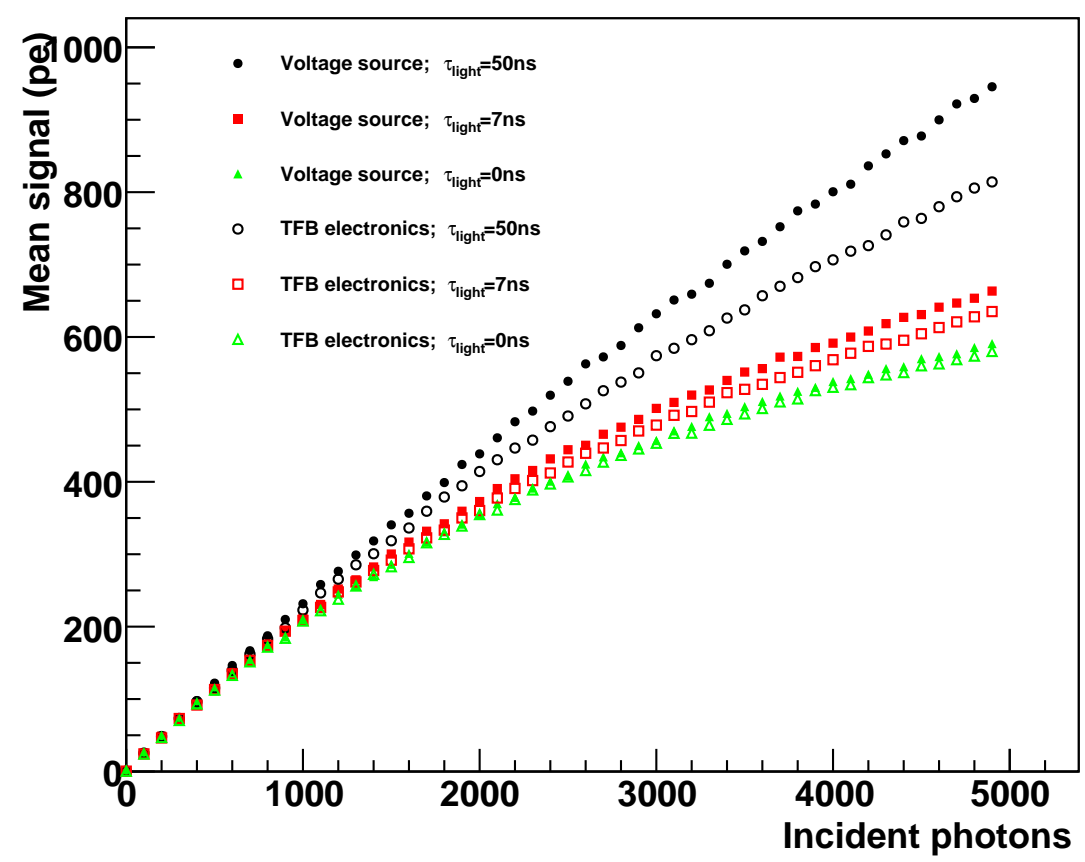

Figure 6: Saturation behaviour predicted by the simulation, for a voltage source recovery model and the ND280 local capacitor model.

\section{References}

[1] R. Terri et al., Status of the T2K Experiment. Proceedings, 10th International Workshop on Tau Lepton Physics (TAU08).

[2] T. Lindner et al., Status of the T2K 280m Near Detector. Proceedings, 34th International Conference on High Energy Physics (ICHEP 2008).

[3] Hamamatsu, MPPC Catalogue (Jul 2007). http://sales.hamamatsu.com/en/products/solid-state-division/si-photodiode-series/mppc.php.

[4] K. Yamamura et al., Products and Development Status of MPPC. Proceedings, International Workshop on New Photon Detectors PD09.

[5] Free Software Foundation, Gnu Scientific Library Reference Manual. http://www.gnu.org/software/gsl/manual.

[6] F. Retiere, Using MPPCs for T2K Near Detector. Proceedings, International Workshop on New Photon Detectors PD09.

[7] D. Orme et al., Measurement of PDE of MPPCs with different wavelengths of light. Proceedings, International Workshop on New Photon Detectors PD09.

[8] A. Vacheret et al., First Results of the Trip-t based T2K Front End Electronics Performance with GM-APDs. Proceedings, International Workshop on New Photon Detectors PD07.

[9] J. Estrada, C. Garcia, B Hoeneison, P. Rubinov, MCM II and the Trip Chip. D0 Note 4009 (2002). 